\title{
Pedagogía no lineal como método de enseñanza de los comportamientos tácticos en los deportes de equipo, aplicación al rugby \\ Non-linear pedagogy as a method of teaching tactical behaviors in team sports, rugby application
} Josep Cantos, Francisco J. Moreno

Universidad Miguel Hernández (España)

Resumen: Desde la pedagogía no lineal se entiende al sujeto como un sistema complejo en continua interacción. La interacción entre los distintos elementos del sistema y el entorno provoca la emergencia de nuevos patrones de comportamiento. Si se introduce una variable externa que altera el equilibrio del sistema, éste adopta un nuevo estado, y esta variable externa se conoce como constreñimiento. Mediante la manipulación de constreñimientos podemos favorecer la aparición de un comportamiento táctico deseado. A la hora de diseñar ejercicios desde esta perspectiva debemos tener en cuenta cuatro principios pedagógicos: complejidad táctica, representatividad de la tarea, exageración y muestreo. En este trabajo se revisan los estudios que abordan el comportamiento táctico desde la pedagogía no lineal, y se discuten los procedimientos para el diseño de ejercicios para el análisis desde esta perspectiva. A partir de los datos mostrados en este estudio se concluye que la pedagogía no lineal puede ser una herramienta útil para el diseño de ejercicios de mejora de los comportamientos tácticos en deportes colectivos.

Palabras clave: Constreñimiento, pedagogía no lineal, rugby, comportamiento táctico, emergencia.

Abstract From non-linear pedagogy the athlete is understood as a complex system in continuous interaction. The interaction between the different elements of the system and the environment causes the emergence of new patterns of behavior. If an external variable is introduced that alters the equilibrium of the system, it adopts a new state, and this external variable is known as constraint. By manipulating constraints, we can favor the appearance of a desired tactical behavior. When designing exercises from this perspective we must consider four pedagogical principles: tactical complexity, task representativeness, exaggeration and sampling. In this paper we review the studies that address tactical behavior from nonlinear pedagogy and discuss the procedures for designing exercises for analysis from this perspective. From the data shown in this study it is concluded that non-linear pedagogy can be a useful tool for the design of exercises to improve tactical behaviors in collective sports.

Keywords: Constraint, non-linear pedagogy, rugby, tactical behavior, emergence.

Pedagogía no lineal, y la teoría de los sistemas dinámicos complejos aplicada al entrenamiento de la táctica deportiva

En los últimos años se han puesto en cuestión las metodologías clásicas para la enseñanza de los movimientos tácticos en los deportes de equipo. Las principales debilidades que se han encontrado sobre este tipo de metodologías son la alta repetitividad de movimientos concretos o el uso de métodos de instrucción directa donde los aprendices se limitan al cumplimiento de las instrucciones proporcionadas por el entrenador no centrándose en el aprendiz, sino en la imitación de patrones (Chow, Davids, Button, y Renshaw, 2015).

Como alternativa a esta metodología, en los últimos años han aparecido otras propuestas entre las que destacamos la pedagogía no lineal, que comparte principios de la teoría de los sistemas dinámicos. Entendemos como un sistema complejo dinámico un organismo que está compuesto de dos o más elementos que interactúan continuamente y tienden hacia el equilibrio. Por ejemplo, un ser humano se compone de millones de células, unos 200 huesos, aproximadamente 600 músculos, diferentes órganos, etc. que interactúan entre sí y con su entorno de forma continua dando lugar a una infinidad de patrones de movimiento observables.

Por ello, se debe entender sujeto y entorno como un todo que está en continua interacción. Como señalan del Villar y González (2014) el sistema deportista-entorno debe entenderse como la verdadera unidad de análisis para entender los distintos comportamientos tácticos que se pueden observar en los deportes colectivos. En esta misma línea, el estudio de Duarte, Araújo, Correia, y Davids (2012) propone el concepto de «super-organismo», con el cual se sugiere la necesidad de considerar las sinergias significativas y las interrelaciones que se producen en los equipos deportivos colectivos como el enfoque de análisis adecuado para la comprensión de los comportamientos tácticos en los deportes colectivos. En este caso no solo se considera el análisis del sujeto con su entorno, sino que se aumenta el análisis a las relaciones entre un conjunto de atacantes y un conjunto de defensores, proponiendo la diada como unidad de análisis. Se entiende como diada aquellos

Fecha recepción: 27-02-18. Fecha de aceptación: 27-05-18

Francisco J. Moreno

fmoreno@umh.es fenómenos que implican a más de un individuo, por ejemplo, las atracciones entre personas, la agresión, la comunicación, etc. Si en alguno de estos procesos solo se estudiara uno de los elementos implicados, se perdería información, ya que los comportamientos de un individuo están influenciados por el comportamiento del otro implicado y viceversa, de ahí la necesidad de estudiar el evento en su totalidad y, si trasladamos esto al deporte, podemos entender la diada como un acoplamiento entre 2 jugadores, aunque podemos entender la diada como el acoplamiento entre más de 2 jugadores (Pic y Navarro, 2017) y en aquellas formadas por atacantes y defensores (Bourbousson, Seve, y McGarry, 2010).

La continua interacción entre elementos del sistema da lugar a la aparición de nuevos patrones de comportamiento. La emergencia de nuevos patrones está causada por cambios en una variable externa (conocido como parámetro de control o constreñimiento) impuesta al sistema que lleva a éste a un estado lejos del equilibrio. Este es el principio fundamental en el que se basa la pedagogía no lineal para la mejora de los procesos de aprendizaje del comportamiento táctico: la manipulación de los constreñimientos como herramienta fundamental para el modelado táctico. Estudios como los de Chow, Davids, Hristovski, Araújo y Passos (2011), Vilar, Araújo, Davids, y Button (2012), Davids, Araújo, Hristovski, Passos, y Chow (2012) o Passos, y Davids (2015) concluyeron que mediante la manipulación de constreñimientos se puede modificar las diferentes conductas y movimientos tácticos que aparecen en las situaciones de juego, facilitando la emergencia de comportamientos tácticos deseados. Bajo esta perspectiva, una mejor comprensión y detección de constreñimientos informacionales clave, permitiría a los entrenadores un mejor análisis del rendimiento deportivo y facilitaría, sobre todo, la planificación y la conducción de los entrenamientos (del Villar Álvarez y González, 2014, Gil, Moreno, Claver, Moreno, y del Villar, 2016).

El cambio observado por la modificación de constreñimientos puede ser explicado por las características y el comportamiento típico de un sistema complejo dinámico. La segunda ley de la termodinámica determina que un sistema tiende hacia el equilibrio. Así, los cambios que se produzcan en el entorno del sistema lo llevarán a un estado «lejos del equilibrio», momento en el cual emergerán fluctuaciones en la dinámica de las relaciones entre los elementos del sistema que lo llevarán a ajustarse a las nuevas condiciones del entorno, mostrando una de las propiedades de los sistemas, la autoorganización (Moreno y Ordoño, 2009) 
hasta adquirir un nuevo estado de equilibrio (Wallace, 1997). Este proceso no tiene por qué estar dirigido ni controlado por ningún agente interno del sistema sino que depende de las características de éste y de las variaciones de su entorno.

Otra de las características de los sistemas dinámicos complejos es la estabilidad que muestren sus patrones de comportamiento. Cada patrón de movimiento que emerge tiene un grado de firmeza o tenacidad que determina su resistencia al cambio. A mayor estabilidad, mayor resistencia al cambio. Otra propiedad remarcable de los sistemas complejos, sobresaliente en los seres vivos, es la habilidad para adaptarse al ambiente y aprender. Se cree que los aprendizajes se realizan a través de la auto-organización en condiciones lejanas del equilibrio. Para conseguir un patrón de comportamiento nuevo se requiere una pérdida de estabilidad del patrón de comportamiento previo y cualquier adaptación se consigue a partir de una experiencia anterior (Moreno y Ordoño, 2009).

La pedagogía no lineal, a diferencia de la metodología clásica, no pretende que los jugadores memoricen diferentes patrones motores concretos para la solución de problemas también muy concretos, que no siempre se reproducen en las situaciones reales de competición. Esta metodología intenta que los jugadores puedan dar solución a los diferentes problemas con distintas soluciones. Pretende dotar a los jugadores de un amplio repertorio motor para dar solución a los distintos problemas que aparecen en el transcurso normal de los deportes colectivos, de una forma mucho más natural. Siguiendo las características principales de esta teoría, los cambios en el comportamiento de los jugadores sería fruto de la capacidad de adaptación del sistema, del que emergen patrones de auto-organización en función de la manipulación de los constreñimientos. Chow et al. (2015), en una revisión sobre este tópico, observaron que una gran cantidad de estudios publicados al amparo de esta teoría dotan a la pedagogía no lineal de un marco teórico suficiente para proponer esta metodología como una herramienta útil para la mejora del aprendizaje de los comportamientos tácticos en los deportes de equipo.

Principios metodológicos de la pedagogía no lineal aplicados a los deportes colectivos

Para la aplicación de la pedagogía no lineal a los deportes colectivos Chow et al., (2015) comentan unos principios pedagógicos que los entrenadores deberían tener en cuenta para el diseño y la planificación de tareas de entrenamiento táctico. Estos principios son: complejidad táctica, representatividad de la tarea, exageración y muestreo.

La complejidad táctica que plantea cada situación de entrenamiento debe estar acorde al nivel de los participantes, es decir, las tareas deben ser adecuadas al grado de competencia de los sujetos que realizan la actividad. Si la complejidad de la tarea es mucho mayor que el nivel de los sujetos, no se facilitará una adaptación a la situación que se plantea. Si por el contrario, la tarea tiene una complejidad mucho menor al grado de competencia de los sujetos, ésta no supondrá ningún reto para los deportistas y por tanto la mejora en el rendimiento deportivo será escasa o nula (Moreno y Ordoño 2009). Así, las tareas deben suponer un reto donde los sujetos puedan descubrir las posibles soluciones al problema planteado. Debe haber un correcto equilibrio entre la complejidad de la tarea planteada y el nivel de los deportistas que realizan el ejercicio. Por ejemplo, si a jugadores de elite en rugby les planteamos una situación de 2 contra 1, donde el defensor no puede desplazarse y por tanto no puede aumentar la presión sobre el jugador atacante, el jugador en posesión del balón superará con mucha facilidad esta situación, y no supondrá una mejora en su rendimiento. Si, por el contrario, en esta misma situación, es un jugador que se está iniciando en este deporte, el simple hecho de tener un contrario delante, y tener que decidir qué tipo de pase realizar, observando al rival y al compañero, puede suponer suficiente estímulo para la mejora del rendimiento de este jugador.

El siguiente principio a tener en cuenta es el muestreo. Dentro de un mismo deporte podemos encontrar ejercicios con características simila- res que se pueden aplicar a diferentes situaciones colectivas para la mejora del rendimiento del comportamiento táctico. Esta similitud también la podemos encontrar en diferentes deportes. Por ejemplo, los deportes colectivos pueden tener características comunes como en los deportes de invasión, los cuales todos comparten el objetivo de conseguir trasladar la pelota al campo rival y anotar. El hecho que ciertos deportes compartan estas características favorece una transferencia de aprendizaje entre deportes, y por ello, se puede exponer a los deportistas a diferentes situaciones y experiencias, que favorezcan la comprensión de los comportamientos tácticos en los distintos deportes colectivos. Los entrenadores pueden crear situaciones de juego muy variadas, para que los jugadores exploren distintas soluciones posibles a los problemas planteados. Por ejemplo, aunque el rugby league, y el rugby tengan normas diferentes, comparten un mismo principio que es avanzar hacia el campo del contrario y conseguir llegar a la zona de anotación para conseguir puntos. De esta forma se pueden utilizar durante entrenamientos ejercicios con las normas de rugby league para poner a los jugadores en situaciones nuevas, y que exploren nuevas soluciones. Estas nuevas experiencias, ampliaran la comprensión de los comportamientos tácticos, ya que estos, aunque son deportes distintos, mantienen los mismos principios.

La representatividad de la tarea se refiere a que aquellas planteadas por los entrenadores deben mantener las características principales del juego real, es decir, debe mantener similitudes básicas con el deporte real en el cual se pretende mejorar el comportamiento táctico. La modificación del número de jugadores o el juego en espacios reducidos puede tener efecto no sólo sobre aspectos fisiológicos (Campos et al. 2017) o motivacionales (Carrasco Chirosa, Tamayo, Cajas y Reigal, 2015), sino que puede ser usado como facilitador de comportamientos tácticos siempre que se mantenga la representatividad. Por ejemplo, se pueden plantear mini-juegos, donde el número de jugadores y espacio es menor a la realidad del deporte en sí, pero la estructura táctica tanto del minijuego como la del deporte real debe ser la misma. Así, se puedemodificar el tamaño de la pelota para facilitar la recepción de esta para jugadores iniciados en el rugby o, por el contrario, podemos disminuir el tamaño del balón para aumentar la coordinación en jugadores más experimentados. Aunque modificamos el tamaño del objeto, no modificamos las demandas tácticas de la tarea.

Por último, mediante el principio de exageración se pretende aumentar o disminuir ciertas características del juego, para facilitar la aparición de diferentes patrones de movimiento o comportamientos tácticos (Tan et al., 2012). En este principio táctico, se postula que la manipulación de constreñimientos, como las dimensiones del campo, u otras variaciones del reglamento (Vizcaíno, Sáenz-López y Rebollo, 2016), puede permitir crear situaciones que favorezcan la aparición de diferentes comportamientos tácticos deseables. Por ejemplo, si mantenemos el número de jugadores, pero aumentamos las dimensiones del campo, estos jugadores tendrán mayor espacio a su alrededor. Este aumento de espacio entre jugadores es una exageración de la situación real durante un partido, que favorece que aparezcan nuevos comportamientos tácticos, como desplazamientos más largos de los jugadores. Podemos modificar el tamaño del campo para aumentar la distancia entre defensores en el rugby, lo cual debe facilitar a los atacantes que penetren por dichos espacios. Por el contrario, podemos disminuir el tamaño del campo para que los defensores dejen menores espacios entre ellos y facilitar que los atacantes deban buscar la defensa y crear una fase contacto con los defensores y proteger la pelota. Dependiendo de los objetivos y los contenidos que desee trabajar el entrenador, puede utilizar este principio para que aquellos aspectos que desea mejorar emerjan de forma natural modificando las condiciones de los ejercicios propuestos.

El entrenador deberá manipular de forma correcta los diferentes constreñimientos para que se favorezca la aparición de los comportamientos tácticos deseables. En el estudio de Serra-Olivares, GonzálezVíllora, García-López, y Araújo (2015) además de señalar cómo la utilización correcta de estos principios pedagógicos es sustentada teóricamente, observaron que la representatividad y la exageración, eran los 
dos principios que más influyen en la aparición de nuevos comportamientos tácticos. El diseño representativo de tareas fundamentado en la problemática táctica se propone, de este modo, como una estrategia efectiva y aplicable en diferentes ámbitos de entrenamiento (SerraOlivares y García-Rubio, 2017). En otro estudio de Chow et al. (2015) consideraban el papel del entrenador como un facilitador de situaciones en las cuales los deportistas tuvieran que resolver problemas tácticos, y la emergencia de patrones tácticos que resolvieran los distintos problemas planteados, y para ello el entrenador debe proponer diferentes ejercicios teniendo en consideración los principios pedagógicos de la metodología no lineal.

El hecho de integrar lo mencionado hasta ahora, facilita que los diseños de procesos de aprendizaje produzcan mayores oportunidades de acción y por tanto un aumento de la capacidad de los sujetos para resolver distintos problemas que se plantean en las actividades. En el estudio de Araújo, et al. (2015) en una de sus conclusiones menciona que se deben diseñar procesos de aprendizaje donde se intente aumentar la variabilidad funcional de los sujetos. Dicho aumento facilita la emergencia denuevos comportamientos. En el estudio de Davids, Button, Araújo, Renshaw, y Hristovski (2006) se resalta el papel de la variabilidad como elemento facilitador de la adaptación, es decir, que el aumento de diferentes situaciones y problemas en entrenamientos del comportamiento táctico favorece la aparición de fenómenos adaptativos capaces de solucionar los distintos problemas planteados en la tarea. Sin embargo, como apunta el trabajo de Passos, Araújo, Davids, y Shuttleworth (2008) no es suficiente con aumentar la variabilidad durante los entrenamientos, ya que se consigue que emerjan distintos nuevos patrones, pero no se consigue la estabilización de ninguno. Así, debe haber un equilibrio preciso entre la variabilidad producida por la incertidumbre que plantea el entorno del sujeto y la estabilidad de las acciones, para que los sujetos puedan afianzar nuevos patrones de movimiento que les permitan satisfacer las necesidades demandadas en las distintas tareas que se puedan plantear.

\section{Principales constreñimientos manipulados para el entrena- miento táctico de los deportes colectivos}

Como se ha mencionado anteriormente, la manipulación de los constreñimientos es una herramienta clave para el modelado de los comportamientos tácticos en deportes de equipo. Los principales constreñimientos analizados en la literatura son los espacio-temporales, los cuales incluyen variables manipulables como velocidad relativa de los jugadores, distancias interpersonales, distancia a la portería, canasta, zona de ensayo, etc. Además, estas variables se han identificado en diferentes deportes colectivos como rugby, futbol y futbol sala o baloncesto. Por ejemplo, en el estudio de Correia et al. (2012) se observó la importancia de la manipulación de constreñimientos espaciotemporales en el diseño de tareas representativas que inducen a la aparición de comportamientos tácticos deseables mediante la modificación de las interacciones entre sujeto y entorno en deportes de equipo. Los trabajos de Rodrigues, y Passos (2013), Passos et al. (2008) y Passos et al. (2009) se centraron en analizar la velocidad relativa y la distancia interpersonal en jugadores de rugby. En estos estudios se observó que estas variables eran importantes porque influyen en la coordinación interpersonal y por tanto favorecen la aparición de nuevas oportunidades de acción. En concreto en el trabajo de Passos et al. (2008) observaron que cuando aparece un nuevo estado en el sistema, en concreto el estado de ensayo, a una distancia de 3'5 metros aparece una disrupción en la coordinación de la diada atacante-defensor, donde el defensor reduce su velocidad y el atacante la aumenta, dando lugar al desequilibrio del sistema y derivando hacia un nuevo estado de equilibrio que en este caso era el ensayo limpio del jugador atacante. Por esto, los entrenadores deben manipular estas dos variables para incentivar la aparición de comportamientos tácticos deseables. La manipulación de estos constreñimientos conduce a la diada atacante-defensor a una situación de inestabilidad que favorece la emergencia de oportunidades de acción y comportamientos tácticos nuevos. Algunos trabajos incluso han en- contrado valores críticos, que llevan al sistema a un estado de inestabilidad y por tanto hacia una nueva forma de relación entre los elementos del sistema. En los estudios de Passos, et al. (2009) y Passos, et al. (2008) encontraron un periodo crítico en la variable de la distancia interpersonal atacante-defensor alrededor de cuatro metros, a partir del que la diada atacante-defensor evolucionó hacia nuevos patrones de coordinación. De una forma similar, se encontró un valor critico en el constreñimiento de la velocidad relativa. En el estudio de Diniz, Barreiros, y Passos (2014) encontraron que el sistema evolucionaba a un estado alternativo a velocidades relativas de $\pm 2 \mathrm{~ms}^{-1}$. Cuando el sistema alcanzaba estas velocidades, entraba en un estado de inestabilidad y hacía que evolucionara a otro estado alternativo. Es decir, en cuanto las velocidades de atacantes y defensores variaban entre ellas $\pm 2 \mathrm{~ms}^{-1}$, si el jugador portador del balón era el que más velocidad llevaba el sistema evolucionaba hacia un nuevo estado donde el jugador atacante sobrepasaba al defensor y conseguía realizar una marca, en cambio cuando ocurría lo contrario, el jugador atacante descendía su velocidad, el sistema evolucionaba hacia otro nuevo estado, donde el jugador defensor conseguía realizar un placaje impidiendo al jugador atacante seguir avanzando.

Dentro de los constreñimientos espacio-temporales, otra variable frecuentemente analizada, es la proximidad a la portería, canasta, zona de ensayo, etc. En los trabajos de Headrick et al. (2012), Travassos, Araújo, Duarte, y McGarry (2012) y Esteves et al. (2012) se observó que la proximidad a la zona de marca influenciaba en el comportamiento emergente de la diada atacante-defensor, y por tanto en el comportamiento táctico. Los entrenadores pueden, mediante la manipulación de esta variable, modelar los comportamientos tácticos deseados. Cuando los jugadores defensores estaban más cerca de su zona de marca, aparecían marcajes más próximos, se reducían la distancia atacante-defensor, los jugadores atacantes cerca de la zona contraria de marca aumentaban la velocidad de los ataques, por tanto la relación entre atacante-defensor se veía modificada en función de la proximidad de la zona de marca.

Otros de los constreñimientos más analizados son el número de jugadores y el tamaño del espacio de juego. La manipulación de estos afectaba a su vez a la variable espacio relativo de cada jugador, que también se ha propuesto como un constreñimiento significativo en el análisis y el entrenamiento táctico. En los trabajos de Silva, et al. (2015) y Silva, Vilar, Davids, Araújo, y Garganta (2016) observaron que la manipulación de estas variables produce la emergencia de nuevas conductas tácticas y por esto concluyeron que puede ser una herramienta útil para el diseño de entrenamientos de mejora de los comportamientos tácticos en deportes de equipo. En el estudio de Silva, et al. (2014) observaron que el aumento de espacio de juego, o la reducción del número de jugadores, aumenta el espacio relativo de cada jugador, lo cual favorece la aparición de comportamientos tácticos con un mayor número de pases largos o en los cuales aparecen mayor número de oportunidades de acción de driblar, realizar un lanzamiento a portería o realizar un pase.

Más allá de constreñimientos espacio-temporales encontramos otros aspectos, como por ejemplo la competitividad, analizada en el estudio de Passos, Araújo, y Davids (2016). La competitividad se puede aprovechar para impulsar procesos adaptativos para la mejora del rendimiento deportivo facilitando las adaptaciones de los jugadores a los distintos problemas planteados en las sesiones de entrenamiento. El liderazgo también fue estudiado como constreñimiento en el trabajo de Passos, Araújo, y Davids (2013). Se observó que puede ser una herramienta válida para la manipulación de comportamientos tácticos intra-equipo, pero también se observó que no lo era para la manipulación de comportamientos tácticos inter-equipo. La utilización de la mano o pie dominante del jugador atacante es otro constreñimiento que se observó en el trabajo de Esteves, et al. (2012) donde se concluyó que la manipulación de este constreñimiento puede facilitar el aprendizaje de los jugadores y mejorar así su rendimiento. En el estudio de Riley, Richardson, Shockley, y Ramenzoni (2011) propuso que la manipulación de la frecuencia de movimiento puede modelar la coordinación rítmica interpersonal y por tanto los patrones de movimiento emergentes. Por último, es necesario indicar que el efecto de la manipulación de 
Tabla 1.

Propuesta de intervención práctica para el diseño de ejercicios que favorezcan el comportamiento táctico del juego en penetración, desplegado y juego al pie. Fase inicial: 2 vs 1.

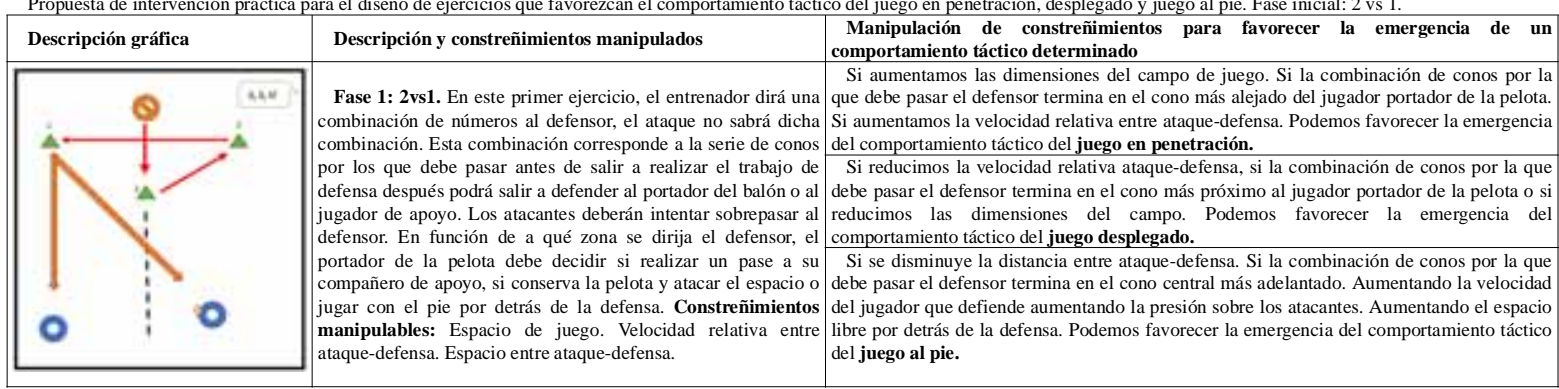

Tabla 2.

Propuesta de intervención práctica para el diseño de ejercicios que favorezcan el comportamiento táctico del juego en penetración, desplegado y juego al pie. Fase segunda: 3 vs

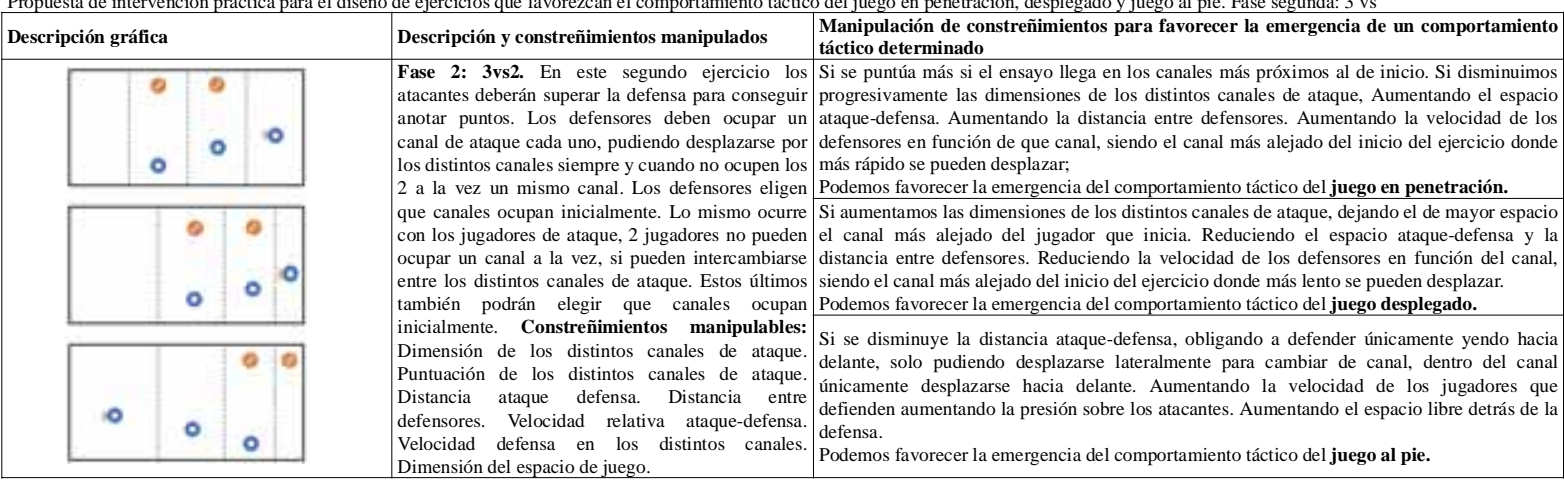

Tabla 3

Propuesta de intervención práctica para el diseño de ejercicios que favorezcan el comportamiento táctico del juego en penetración, desplegado y juego al pie. Fase tercera: 4 vs 1 / 2 / 3 .

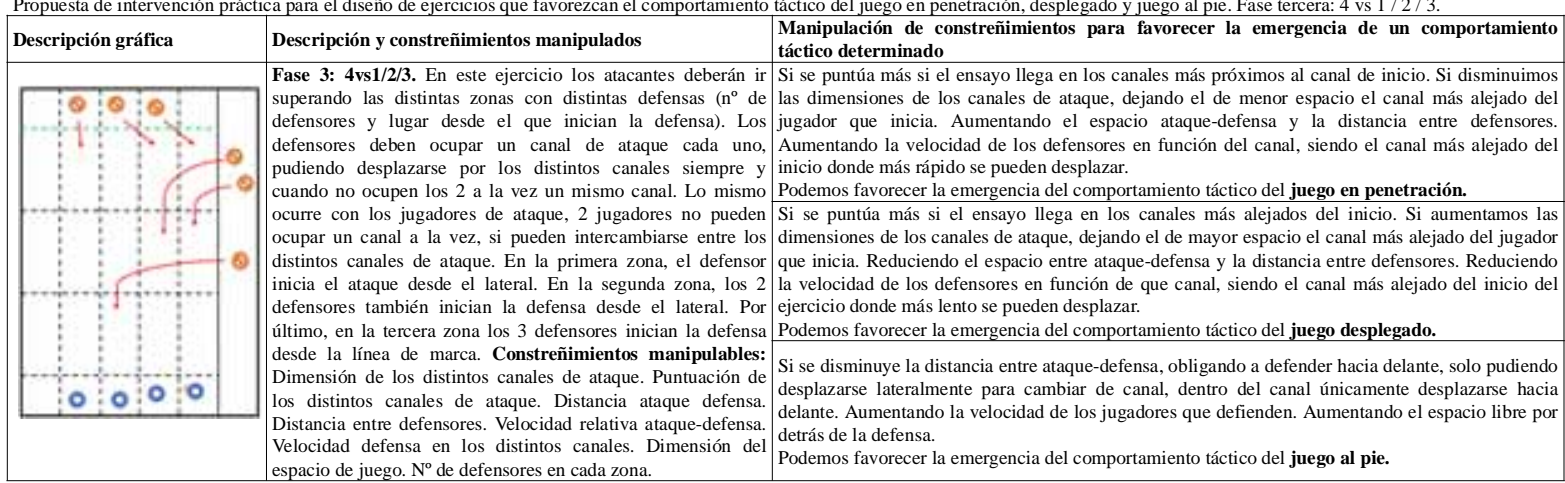

constreñimientos para facilitar la emergencia de determinados comportamientos tácticos puede estar mediado por el nivel de habilidad y las capacidades individuales de los jugadores y por tanto se debe tener en cuenta a la hora del diseño de programas de entrenamiento Silva, et al. (2014).

\section{Aplicaciones y conclusiones}

Como ejemplo de aplicación de los contenidos expresados anteriormente se expone una propuesta práctica concreta ejemplificada en tres fases. En una primera fase (tabla 1) se propone manipular los constreñimientos de espacio total de juego, velocidad relativa y el espacio entre ataque-defensa, para favorecer la emergencia de diferentes comportamientos tácticos. Los comportamientos tácticos propuestos en el ejemplo son el juego en penetración, el juego desplegado y el juego al pie. Así, la manipulación de las diferentes combinaciones de constreñimientos en los ejercicios favorecería la emergencia de un comportamiento táctico u otro, tal y como se comenta en la propuesta. En la segunda propuesta, o fase 2 (tabla 2), se añade la manipulación de la dimensión de los distintos canales de ataque así como la velocidad de la defensa en dichos canales. Porúltimo, con el objetivo de que los atacantes deban ir superando las distintas zonas con distintas defensas $\left(\mathrm{n}^{\circ}\right.$ de defensores y lugar desde el que inician la defensa), se propone un tercer nivel de complejidad táctica en la tercera fase de aplicación práctica (tabla 3). En esta propuesta se incrementan las posibilidades de actuación y se manipula la velocidad de la defensa en los distintos canales de desplazamiento, así como los constreñimientos comentados en las fases anteriores.

En este trabajo hemos argumentado por qué la pedagogía no lineal puede ser una buena herramienta para el diseño de ejercicios centrados en la mejora de los comportamientos tácticos en deportes colectivos. Hemos observado como esta metodología está respaldada por la literatura científica y se considera una herramienta válida para el aprendizaje de comportamientos tácticos. A la hora de diseñar los ejercicios debemos tener en cuenta que cumplan con los principios pedagógicos de esta metodología: complejidad táctica, representatividad de la tarea, exageración y muestreo. Además, cuando analizamos los comportamientos que emergen durante los ejercicios debemos considerar la diada como elemento de análisis aproximándonos desde una perspectiva multinivel para una correcta comprensión de los distintos comportamientos que se dan durante los ejercicios. La principal herramienta de esta pedagogía es la manipulación de los constreñimientos o parámetros de control que afectan al sistema complejo para favorecer la emergencia de los comportamientos tácticos deseados. La correcta manipulación de ciertos constreñimientos puede favorecer la emergencia de un comportamiento táctico u otro. El parámetro de control, o constreñimiento, no aporta información sobre la naturaleza de los cambios de patrones, pero conduce al sistema a través de varios patrones que son el resultado de las propiedades de auto-organización de un sistema lejos del equilibrio (Capra, 1998). Gracias a la manipulación de situaciones de juego el entrenador debe ser capaz de generar situaciones que favorezcan la emergencia de los comportamientos tácticos deseables sin la necesidad 
de realizar ejercicios repetitivos y sin tener que dar directamente la solución a los jugadores. Son los jugadores quienes encontraran la mejor solución, y el entrenador debe manipular los constreñimientos para que la solución más probable se dirija hacia los objetivos que se deseen.

\section{Líneas de investigación futuras}

Una vez revisados los aspectos más relevantes de la perspectiva no lineal aplicada al entrenamiento táctico en los deportes colectivos, con aplicación al rugby, se abren nuevas vías de investigación futura que pueden aportar nuevos conocimientos y procedimientos tanto de entrenamiento como de análisis del comportamiento táctico. Así, una de las posibles futuras líneas de investigación sobre esta temática debería orientarse sobre las herramientas más adecuadas para medir tanto el comportamiento táctico como la mejora de rendimiento táctico en deportes colectivos. Se conocen algunas herramientas que son válidas para medir ciertos componentes que intervienen en las diadas que son analizadas, pero se necesitan más trabajos que analicen los distintos métodos de medición y determinar cuáles son los más precisos o más efectivos para medir mejora del rendimiento en los comportamientos tácticos de los deportes colectivos.

De cualquier modo, la principal línea de investigación futura procedente de las conclusiones vertidas en este trabajo debe corresponderse con llevar a la práctica las propuestas de actuación elaboradas a la luz de la teoría que apoya la utilización de la pedagogía no lineal en los deportes colectivos. Se debe progresar en el conocimiento de esta línea de investigación a través de la aplicación de programas de mejora de los comportamientos tácticos en deportes colectivos basados en la pedagogía no lineal y comprobar de manera empírica la aplicación de estos. Algunos trabajos, como se han mencionado a o largo del texto, han propuesto intervenciones pero pocos de ellos muestran resultados concluyentes de trabajos experimentales y muy pocos han mostrado su efecto a medio o largo plazo en la formación de los deportistas. Deben llevarse a cabo estudios en esta línea de investigación para contrastar los postulados propuestos y hayar la mejor forma de aplicación práctica.

\section{Referencias}

Araújo, D., Passos, P., Esteves, P., Duarte, R., Lopes, J., Hristovski, R., \& Davids, K. (2015). The micro-macro link in understanding sport tactical behaviours: Integrating information and action at different levels of system analysis in sport. Movement \& Sport Sciences, (3), 53-63.

Araújo, D., Silva, P., \& Davids, K. (2015). Capturing group tactical behaviors in exper team players. In D. Farrow (Ed.) Rotledge Handbook of Sport Expertise: Routledge.

Bourbousson, J., Seve, C., \& McGarry, T. (2010). Space-time coordination dynamics in basketball: Part 1. Intra-and inter-couplings among player dyads. Journal of sports sciences, 28(3), 339-347.

Bourbousson, J., Seve, C., \& McGarry, T. (2010). Space-time coordination dynamics in basketball: Part 2. The interaction between the two teams. Journal of Sports Sciences, 28(3), 349-358.

Bourbousson, J., Deschamps, T., \& Travassos, B. (2014). From players to teams: towards a multi-level approach of game constraints in team sports. International Journal of Sports Science \& Coaching, 9(6), 1393-1406.

Carrasco Beltrán, H., Chirosa Ríos, L., Tamayo, I., Cajas Luna, B., \& Reigal Garrido, R. (2015). Efectos de un programa extraescolar basado en juegos reducidos sobre la motivación y las necesidades psicológicas básicas en las clases de educación física. Revista Iberoamericana de Psicología del Ejercicio y el Deporte, 10 (1), 23-31.

Campos Vázquez, M., Casamichana Gómez, D., Suárez Arrones, L., González Jurado, J. Toscano Bendala, F., \& León Prados, J. (2017). Medium-sided games in soccer: physical and heart rate demands throughout successive working periods. Journal of Human Sport and Exercise, 12 (1), 129-141.

Chow, J. Y., Davids, K., Hristovski, R., Araújo, D., \& Passos, P. (2011). Nonlinear pedagogy: Learning design for self-organizing neurobiological systems. New Ideas in Psychology, 29(2), 189-200.

Chow, J. Y., Davids, K., Button, C., \& Renshaw, I. (2015). Relations between nonlinea pedagogy and games-based teaching approaches. In K. Davids. et al. (Eds.) Nonlinear pedagogy in skill acquisition: An introduction (pp. 152-165). Routledge.

Chow, J. Y., Davids, K., Button, C., \& Renshaw, I. (2015). Why do we what we do?. In K. Davids et al. (Eds.) Nonlinear pedagogy in skill acquisition: An introduction (pp. 166-184). Routledge.

Correia, V., Araujo, D., Craig, C., \& Passos, P. (2011). Prospective information for pass decisional behavior in rugby union. Human Movement Science, 30(5), 984-997.

Correia, V., Araújo, D., Duarte, R., Travassos, B., Passos, P., \& Davids, K. (2012). Changes in practice task constraints shape decision-making behaviours of team games players. Journal of Science and Medicine in Sport, 15(3), 244-249.

Davids, K., Button, C., Araújo, D., Renshaw, I., \& Hristovski, R. (2006). Movement models from sports provide representative task constraints for studying adaptive behavior in human movement systems. Adaptive behavior, 14(1), 73-95.

Davids, K., Araújo, D., Hristovski, R., Passos, P., \& Chow, J.Y.(2012). Ecological dynamics and motor learning design in sport. Skill acquisition in sport: Research, theory \& practice, $112-130$

del Villar Álvarez, F., \& González, L. G. (2014). La manipulación de los constreñimientos en deportes de equipo. El entrenamiento táctico y decisional en el deporte (pp. 195-208).

del Villar Álvarez, F., \& González, L. G (2014). La toma de decisiones en el deporte bajo la perspectiva del sistema individuo-entorno. El entrenamiento táctico y decisional en el deporte (pp. 43-58).

Diniz, A., Barreiros, J., \& Passos, P. (2014). To Pass or Not to Pass: A Mathematical Model for Competitive Interactions in Rugby Union. Journal of motor behavior, 46(5), 293-302.

Duarte, R., Araújo, D., Correia, V., \& Davids, K. (2012). Sports teams as superorganisms. Sports medicine, 42(8), 633-642.

Esteves, P. T., Araújo, D., Davids, K., Vilar, L., Travassos, B., \& Esteves, C. (2012) Interpersonal dynamics and relative positioning to scoring target of performers in 1 vs. 1 sub-phases of team sports. Journal of sports sciences, 30(12), 1285-1293.

Gil, A., Moreno, P., Claver, F., Moreno, A., \& del Villar, F. (2016). Manipulación de los condicionantes de la tarea en Educación Física: Una propuesta desde la pedagogía no lineal. Retos, (29), 22-27

Headrick, J., Davids, K., Renshaw, I., Araújo, D., Passos, P., \& Fernandes, O. (2012). Proximity-to-goal as a constraint on patterns of behaviour in attacker-defender dyads in team games. Journal of sports sciences, 30(3), 247-253.

Hristovski, R., Davids, K., Araujo, D., \& Passos, P. (2011). Constraints-induced emergence of functional novelty in complex neurobiological systems: a basis for creativity in sport. Nonlinear Dynamics-Psychology and Life Sciences, 15(2), 175.

Passos, P., Araújo, D., Davids, K., Gouveia, L., Milho, J., \& Serpa, S. (2008). Informationgoverning dynamics of attacker-defender interactions in youth rugby union. Journal of Sports Sciences, 26(13), 1421-1429.

Passos, P., Araújo, D., Davids, K., \& Shuttleworth, R. (2008). Manipulating constraints to train decision making in rugby union. International Journal of Sports Science \& Coaching, 3(1), 125-140.

Passos, P., Araujo, D., Davids, K. W., Gouveia, L., Milho, J., \& Serpa, S. (2009). Interpersonal coordination tendencies, decision-making and information governing dynamics in rugby union. In D. Araujo et al. (Eds) Perspectives on Cognition and Action in Sport (pp. 27-42). Nova Science Publishers, Inc.

Passos, P., Araújo, D., \& Davids, K. (2013). Self-organization processes in field-invasion team sports. Sports Medicine, 43(1), 1-7.

Passos, P. L., \& Davids, K. (2015). Learning design to facilitate interactive behaviours in Team Sports. RICYDE. Revista Internacional de Ciencias del Deporte, 11(39), 18-32.

Passos, P., Araújo, D., \& Davids, K. (2016). Competitiveness and the Process of Coadaptation in Team Sport Performance. Frontiers in psychology, 7.

Pic Aguilar, M. \& Navarro Adelantado, V. (2017). Triad Communication and Specificity of Motor Games. Revista Internacional de Medicina y Ciencias de la Actividad Física y el Deporte vol. 17 (67) pp. 523-539.

Riley, M. A., Richardson, M., Shockley, K., \& Ramenzoni, V. C. (2011). Interpersonal synergies. Frontiers in psychology, 2, 38.

Rodrigues, M., \& Passos, P. (2013). Patterns of Interpersonal Coordination in Rugby Union: Analysis of Collective Behaviours in a Match Situation. Advances in Physical Education, 3(04), 209.

Sampaio, J., \& Maçãs, V. (2012). Measuring tactical behaviour in football. International Journal of Sports Medicine, 33(05), 395-401.

Serra-Olivares, J., \& Garcia-Rubio, J. (2017). Tactical problems, key component of the representative design of tasks within the non-linear pedagogy approach applied to sports. Retos, 32, 270-278.

Serra-Olivares, J., González-Villora, S., García-López, L. M., \& Araújo, D. (2015). Gamebased approaches' pedagogical principles: exploring task constraints in youth soccer. Journal of human kinetics, 46(1), 251-261.

Silva, P., Aguiar, P., Duarte, R., Davids, K., Araújo, D., \& Garganta, J. (2014). Effects of pitch size and skill level on tactical behaviours of Association Football players during small-sided and conditioned games. International Journal of Sports Science \& Coaching, 9(5), 993-1006.

Silva, P., Duarte, R., Sampaio, J., Aguiar, P., Davids, K., Araújo, D., \& Garganta, J. (2014). Field dimension and skill level constrain team tactical behaviours in small-sided and conditioned games in football. Journal of sports sciences, 32(20), 1888-1896.

Silva, P., Esteves, P., Correia, V., Davids, K., Araújo, D., \& Garganta, J. (2015). Effects of manipulations of player numbers vs. field dimensions on inter-individual coordination during small-sided games in youth football. International Journal of Performance Analysis in Sport, 15(2), 641-659.

Silva, P., Duarte, R., Esteves, P., Travassos, B., \& Vilar, L. (2016). Application of entropy measures to analysis of performance in team sports. International Journal of Performance Analysis in Sport, 16(2), 753-768.

Silva, P., Vilar, L., Davids, K., Araújo, D., \& Garganta, J. (2016). Sports teams as complex adaptive systems: manipulating player numbers shapes behaviours during football small-sided games. SpringerPlus, 5(1), 191.

Travassos, B.,Araújo, D., Duarte, R., \& McGarry, T. (2012). Spatiotemporal coordination behaviors in futsal (indoor football) are guided by informational game constraints. Human Movement Science, 31(4), 932-945.

Verdú, N.P., Ariño, D.G., \& Martínez, J.A.C. (2017). Análisis comparativo de la metodología mixta y la basada en juegos reducidos en el fútbol base. Retos, 32, 199-203

Vilar, L., Araújo, D., Davids, K., \& Button, C. (2012). The role of ecological dynamics in analysing performance in team sports. Sports Medicine, 42(1), 1-10.

Vizcaíno, C.; Sáenz-López, P. y Rebollo, J.A. (2016). Relationship Between Rules and Technical and Tactical Contents in Minibasket. Revista Internacional de Medicina y Ciencias de la Actividad Física y el Deporte, 16 (64), 807-823 